\title{
Febrile Seizure Detect and Alarm System
}

\author{
http://dx.doi.org/10.3991/ijes.v3i4.4332 \\ Khader Mohammad, Sundous Hussein, Rawan Rimawi \\ Birzeit University, Ramallah, Palestine
}

\begin{abstract}
Wearable medical technology is becoming increasingly popular. Such devices have the potential to vastly improve the effectiveness, safety and economy of monitoring vital signs including temperature by both patients and clinicians. The purpose of this paper is to present design and implementation of a reliable, low cost and non-intrusive system that is to monitor a child's body temperature using high precision temperature sensor and alarm his/her parents or guardian on their mobile phones as well as on a webpage which can be accessed via internet connection so as to avoid convulsions brought on by a significant rise in body temperature.
\end{abstract}

The system provides a low power reliable, non-intrusive and noninvasive body temperature monitor that processes and analyses data acquired from sensors designated to monitor body temperature and limbs movements. The sensors interfaced to communicate with a mobile application that is installed on the child's guardian mobile and with a webpage so as to provide remote monitoring as well as issuing an alarm of febrile seizure occurrence.

This research measure temperature using a device that can be worn as a watch, which is arguably more comfortable than other locations (mouth, ear and rectum). Measuring body temperature can be very challenging as it depends on factors including level of activity, time of the day as well as psychological factors.

The theory, design procedures and discussions are presented in this research. High accuracy rate of $\pm 1 \% \mathrm{C}^{\circ}$ is achieved with cost of $185 \$$. The system has been prototyped and tested.

Index Terms-Febrile Seizure Detect and Alarm System, Healthcare Systems, Infant's Fever, Remote Temperature Monitoring.

\section{INTRODUCTION}

eFbrile seizures are convulsions brought on by a fever in infants or small children. Approximately, one in every twenty five children will have at least one febrile seizure. A few factors appear to boost a child's risk of having recurrent febrile seizures, including young age (less than 15 months) during the first seizures, frequent fevers and having immediate family members with a history of febrile seizures.

Febrile seizures don't cause brain damage, mental retardation or learning disabilities. However, during a seizure, there is a chance that the child may be injured by falling or may choke from food or saliva in the mouth. Proper detection and alarm aid systems for seizures can help avoid these hazards.

Studies by the Central Bureau of Statistics indicate that spending on healthcare has significantly increased between the years 2000 and 2008 [1]. Statistics suggest that the spending as well as the cost of healthcare systems and medical goods dedicated to monitoring indicators of child health care are notably increasing. This clearly indicates a tremendous need for low cost, reliable and non-intrusive systems that provide a proper mechanism to monitor the healthcare indicators of children. One health care indicator of interest is temperature; an increase in body temperature might lead to what so called febrile seizure.

Systems that allow monitoring of children's vital signs including body temperature are readily available in the market. However, none of them is designed to detect febrile convulsions while interfaced to communicate with a mobile phone for remote monitoring. The objective of this paper is to present such a system. A proper detection and alarm mechanism for detecting febrile convulsions by means of sensors is the main goal of this paper. The system is meant to: accurately measure body temperature, alarm the child's guardian when a certain temperature limit is reached, and transmit temperature readings acquired by sensors to the mobile of the child's guardian wirelessly and continuously record temperature readings for a period of up to 48 hours. Since available systems provide no wireless monitoring mechanism. There is a need for such a device to provide warning messages to parents or caregivers before the febrile convulsion begins, and it is very desirable if such a device were small size for wearing on arm, hand or leg.

This research intended to provide a low power reliable, non-intrusive and noninvasive body temperature monitor that processes and analyses data acquired from sensors designated to monitor body temperature and limbs movements. The sensors will be interfaced to communicate with a mobile application that is installed on the child's guardian mobile to provide remote monitoring as well as issuing an alarm of febrile seizure occurrence on the monitor as well as the mobile phone.

Body temperature can be measured from different locations of the body. This research intend to measure temperature using an underarm sensor as it is arguably more comfortable than other locations (mouth, ear and rectum). Measuring body temperature can be very challenging as it depends on factors including: level of activity, time of the day as well as psychological factors.

The rest of the paper is structured as follows: section II cover the literature reviews followed by the proposed system hardware and software requirements.

\section{LITERATURE REVIEW}

Traditionally mercury thermometers have been used to record body temperature. However mercury is highly toxic and tends to remain in the environment. Thus, disposal is a serious problem. Mercury thermometers have been successfully replaced with digital alternatives. Still, reliability 
as well as accuracy are issues to be addressed in digital thermometers. According to Liverpool University Institute of Child Health Researchers, digital thermometers sometimes do not register fevers at all and can have one or two degrees difference compared to rectal or oral readings [2].

Recently, several electronic systems have been developed to monitor body temperature as well as other vital signs - Pulse Rate, Blood Temperature, Body Temperature and Breathing Rate. Such systems include: Gastrointestinal Temperature Measurement, MSR Wireless Temperature Monitor, Wireless LifeGuard and Spot Vital Signs LXi.

\section{A. Gastorintestinal Temp. Measurement [3]:}

Gastrointestinal temperature is measured by ingesting a telemetric temperature sensor that transmits the temperature of the GI environment wirelessly to an external logger. Telemetric temperature measurement was first used in animal studies. The use of a telemetric temperature sensor to measure GI temperature in humans was reported only about 10 years ago. GI temperature measurement provides an acceptable level of accuracy without causing discomfort to the user. Thermoregulatory and during sleep research as well as military are key use cases for which such system was originally designed.

\section{B. Msr Wireless Temperature Monitor [4]:}

MSR Wireless Temperature Monitor is a device intended for remote monitoring of body temperature for up to 100 hours of continuous use. The device transmits readings effectively up to 160 feet distance (approx. 50M) in an open source. If there are objects in that space, the transmission distance will be affected. Unlike our proposed system, MSR Wireless Temperature Monitor provides no remote monitoring via mobile phone. MSR Wireless Temperature Monitor transmits readings effectively up to 160 feet distance while the proposed monitor will be configured to transmit up to 300 feet distance.

\section{Wireless Lifeguard[5]:}

Wireless LifeGuard, is a lightweight, portable device developed by NASA scientists to enable monitoring health of astronauts during space travel, it was developed for astronauts, but has many potential uses in clinical, homehealth and military applications as well.

\section{Spot Vital Signs Lxi [6]:}

Spot Vital Signs LXi, measures systolic and diastolic pressure, pulse rate, temperature (oral, adult auxiliary, pediatric auxiliary, rectal, and ear), and pulse oximetry ( $\mathrm{SpO} 2)$ as well as calculates Mean Arterial Pressure. Furthermore, Spot Vital Signs LXi allows the entry of height, weight, respiration rate, and pain level. It also calculates Body Mass Index (BMI) following height and weight entry. The device is intended to be used by clinicians and medically qualified personnel.

The major disadvantages of briefly descried systems above include: intended to be used by clinicians and medically qualified personnel, originally designed for thermoregulatory, during sleep research, relatively expensive and most importantly provide no remote monitoring, Thus, we intend to provide a cost-efficient and easy to use device for regular people that accurately monitors body temperature and issues an alarm when a certain limit is reached.
In this research, the proposed system is to be placed on the child's arm to monitor his/her temperature and limbs movements. An alarm will be issued if the temperature exceeds a certain limit and if violent frequent limbs movements were detected.

\section{Proposed System Hardware AND SOFTwARE REQUIREMENTS}

This section provides a description of hardware and software requirements of the system and how they are integrated to achieve the intended goals. A system functional block diagram is shown in Figure 1.

Temperature and motion sensors will be used. A timer will be triggered as the seizure begins to measure the duration of the seizure, since seizures that last for more than five minutes require immediate treatment and can be harmful.

Temperature and motion sensors are to be interfaced with a microprocessor in which temperature readings will be compared with a threshold. Temperature readings as well as an alarm signal will be transmitted to the mobile phone of the child's guardian if such readings exceeded the predefined threshold. To insure accuracy three successive readings exceeding the threshold will be required to trigger the alarm process. The system flowchart is shown in Figure 2.

Subsections below describe in details the selection process of the appropriate physical elements that comprise the system.

\section{A. Hardware Requirement}

\section{Arduino UNO}

Router

XBEE

Mobile Phone

ATMEGA 328

\section{XBEE}

Figure 1.

Hardware diagram of the system is shown in Figure 1. High Precision Thermistor (MF51E) will be used to supply temperature readings. Such readings will be acquired using ATmega328 and transmitted wirelessly from one XBee module stationed on the Child's arm to another stacked on top of an Arduino Uno board. An Ethernet Shield stacked on top of an Arduino Uno will provide internet connectivity. Thus, data would be transmitted over Internet to the mobile of the Child's guardian.

\section{1) Sensors}

Choosing an appropriate temperature sensor necessitates prioritizing system design attributes. In an attempt to measure the external human body temperature, the following factors are of great importance: Fast Response Time, High Sensitivity and Low Cost.

Constantly measuring body temperature in order to prevent any increase above the normal range requires using a sensor that needs a minor time to change its output from 
its previous state to a new final settled value (Fast Response Time). Such sensor is required as well to be Highly Sensitive that is the minimum change in skin temperature yields a detectable output. Using a Low Cost sensor contributes to the reduction of the overall cost of the system. Thus, the system would suite the purchasing power of average customers.

Factors of great importance will be assigned higher weights than those of less importance. As stated earlier; Fast Response Time, High Sensitivity and Low Cost are the most important factors in our sensor selection process. Table 1 maps different factors of relevance importance to weights. Table 2 below compares characteristics of different existing temperature sensors. Table 3 is constructed with reference to Table 2. Each Temperature Sensor is assigned a value from $1-5$ ( 5 being the highest) for each factor. Such value depends on the characteristics of the Temperature Sensor listed below in Table 1. The Total Score of a Temperature Sensor being the sum of factors weight multiplied by values assigned earlier.

As Table 3 indicates; the thermistor is the temperature sensor that best meets our system requirements. The next section considers different types of thermistors and chooses among them.

\section{2) Thermistor}

The term thermistor is a contraction of the words thermal and resistor. A thermistor's resistance varies significantly with temperature and is composed of a sintered semiconductor material which exhibits a large change in resistance proportional to a small change in temperature. Thermistors belong to the class of absolute-temperature sensors; they can measure temperature that is referenced to an absolute-temperature scale [8]. All thermistors are divided into two groups:

1. Negative Temperature Coefficient Thermistor (NTC): NTC thermistors exhibit a decrease in their electrical resistance when subjected to an increase in temperature. Thus, the temperature is inversely proportional to the resistance. Figure 4 shows the typical non-linear relation between the NTC thermistor's resistance and temperature

2. Positive Temperature Coefficient Thermistor (PTC): PTC thermistors exhibit an increase in their electrical resistance when subject to an increase in temperature.

NTC and PTC thermistors make use of the characteristics inherent in their composition. This qualifies each of them for a certain set of applications. One major application of NTC thermistors is Temperature Measurement and Control. Thus, the proposed system we will use an NTC type thermistor to provide temperature readings.

Changes in the resistance of the NTC thermistor can be brought about either externally by a change in ambient temperature or internally by self-heating resulting from a current flowing through the device. All practical applications are based on this behavior [13]. The high sensitivity and low cost of an NTC thermistor makes it an ideal candidate for precision temperature sensing.

Three different NTC thermistors are NTC 400 Series Thermistors, NTC MA Series Thermistors and NTC MF Series Thermistors.

The Table indicates that 427 Reusable Skin Probe is relatively expensive. Since we strive to provide a device that suits the purchasing power of an average individual, 427
Reusable Skin Probe was eliminated. MF51E High Precision Thermistor is better than MA-300 Reusable Skin Thermistor in terms of tolerance as well as price. Thus, MF51E High Precision Thermistor will be used to supply temperature readings.

Precision Temperature Measurement applications are granted when an NTC thermistor is configured to operate in Resistance Versus Temperature mode. Therefore, MF51E High Precision Thermistor will be configured to operate in this mode. To use a thermistor, its transfer function - Temperature Dependence of a Resistance - must be accurately established. Several mathematical models of a thermistor transfer function have been proposed:

Steinhart-Hart Equation: This equation is a third order polynomial that provides excellent curve fitting for specific temperature spans within the temperature range of $-80{ }^{\circ} \mathrm{C}$ to $260{ }^{\circ} \mathrm{C}$ [20]. The mathematical expression is as follows:

$$
\begin{gathered}
\left.\frac{1}{\mathrm{~T}}=\mathrm{A}+\mathrm{B} \times \ln (\mathrm{R})+\mathrm{c} \times \ln ^{3}(\mathrm{R}) \rightarrow \text { (Equation } 1\right) \\
\text { Where; }
\end{gathered}
$$

A, B and C are the Steinhart - Hart parameters

$\mathrm{R}$ is the thermistor resistance $\Omega$

$\mathrm{T}$ is thermistor temperature $\mathrm{K}$

B Parameter Equation [21]:If a thermistor's resistance $\mathrm{R} 0$ at a calibrating temperature T0 is known; then the Resistance - Temperature relationship is expressed as:

$$
\begin{gathered}
\mathrm{R}=\mathrm{R}_{0} \times \mathrm{e}^{\beta \times\left(\frac{1}{\mathrm{~T}}-\frac{1}{\mathrm{~T}_{0}}\right)} \rightarrow \text { (Equation 2) } \\
\text { Where; }
\end{gathered}
$$

$\mathrm{R}$ is the thermistor resistance $\Omega$ at temperature T K

$\beta$ is the material characteristic temperature $\mathrm{K}$

Table 4 compares the two models listed above. It indicates that Steinhart - Hart Equation provides higher accuracy than $\beta$ Parameter equation. Since accuracy is a factor of great importance, Steinhart - Hart Equation will be used to model the thermistor transfer function.

Using Steinhart - Hart Equation produces a nonlinear output. Thus, hardware linearization is needed.

One of the most commonly used circuits for the purpose of NTC thermistor linearization is the voltage divider circuit, shown in Figure 5.

A series resistor Rs is added to the NTC thermistor resistance Rntc. The output voltage Vout is taken to be around the series resistor and is given by:

$$
\left.\mathrm{V}_{\text {out }}=\frac{\mathrm{R}_{\mathrm{s}}}{\mathrm{R}_{\mathrm{s}}+\mathrm{R}_{\mathrm{ntc}}} \times \mathrm{V}_{\text {in }} \rightarrow \text { (Equation } 3\right)
$$

When an NTC thermistor measures low temperatures; its resistance significantly increases - Negative Temperature Coefficient -. Therefore, the NTC resistor Rntcwill be notably higher than the series resistor and the output voltage is

$$
\mathrm{V}_{\text {out }}=\frac{\mathrm{R}_{\mathrm{s}}}{\mathrm{R}_{\mathrm{ntc}}} \times \mathrm{V}_{\text {in }} \rightarrow \text { (Equation 4) }
$$

The series resistor Rs is eliminated from the denominator. Since Rntc $>>$ Rs; Vout $\cong 0$ Volt.

Similarly, when an NTC thermistor measures high temperatures; its resistance significantly decreases. Therefore, the NTC resistor Rntc will be notably smaller than the series resistor and the output voltage is: 


$$
\mathrm{V}_{\text {out }}=\frac{\mathrm{R}_{\mathrm{s}}}{\mathrm{R}_{\mathrm{s}}} \times \mathrm{V}_{\text {in }} \rightarrow \text { (Equation 5) }
$$

The NTC thermistor resistor Rntcis eliminated from the denominator. Since Rntc $<<$ Rs; Vout $\cong$ Vin.

The previously described extreme cases clarify how the linearization occurs; where the output voltage values vary within the range [0 - Vin] Volts.

For more accurate results, mathematical curve fitting techniques would be applied. An approximately linear relationship is shown in Figure 6.

\section{3) Microprocessor - Arduino Uno}

Arduino is an open source electronics prototyping platform based on flexible, easy to use hardware and software. Arduino can sense the environment by receiving input from a variety of sensors and can affect its surroundings by controlling lights, motors and other actuators.

Parallax Basic Stamp, Net media's BX-24, Phidgets, MIT's Handyboard are microcontrollers and microcontrollers platform that offer similar functionality to Arduino. However, Arduino offers some advantages over such systems as mentioned in [22]. Arduino Unowas selected. An Arduino Uno - R3 board is used to acquire data from temperature sensors. For more information about the Arduino Uno see [24].

\section{4) Transmitter - Xbee Modul}

XBee RF modules are embedded solutions providing wireless end point connectivity to devices. These modules use the IEEE 802.15.4 networking protocol for fast point to multipoint or peer to peer networking. They are designed for high throughput applications requiring low latency and predictable communication timing [25]. It provides up to $250 \mathrm{Kbps}$ of data throughput between nodes on a CSMA/CA network.

In comparison to Bluetooth (IEEE 802.15.1), the LRWPAN is designed as a much simpler protocol with lower data transfer rates (250 kbps compared to $1 \mathrm{Mbps}$ ). Bluetooth was designed as a replacement for peripheral cables and is used in communications between handheld devices, such as phones, requiring access security and high rates of data transfer [26].

Series 1 - 802.15.4 modules - and Series 2 - ZB modules - are quite similar. However, selection of a module is based on application specific needs. [27] Compares specification of four different variants of the two series - Pro and Non-Pro. The XBee ZB modules extend the range of the network through routing. They form self-establishing and self-healing networks for moving data across the network. ZB modules improve on the power output and data protocol. Such modules allow creating complex mesh networks based on the XBee ZB ZigBee mesh firmware.

Two XBee PRO $60 \mathrm{~mW}$ 802.15.4 modules were selected. One stationed on the child's arm is to transmit data acquired from sensors to the XBee module stacked on top of Arduino Uno.

\section{5) Arduino Xbee Shield}

Arduino XBee shield, mates with Arduino, and equips it with wireless communication capabilities using XBee modules including: Series 1, Series 2 as well as Series 2.5, standard and Pro version. An on-board switch labeled Serial Select allows the wireless module to communicate with the USB-to serial converter chip or with the microcontroller.
When in MICRO position, DOUT pin of the XBee module is connected to RX pin of the microcontroller; and DIN is connected to TX. The wireless module will then communicate with the microcontroller. When in USB position, DOUT pin of the XBee module is connected to RX pin of the USB-to-serial converter; and DIN is connected to TX pin of the USB-to-serial converter. Thus, the module communicates directly with the computer. The microcontroller on the board will be bypassed. The microcontroller must be programmed with an empty sketch if the shield is to be used in this mode [28].

An XBee shield will be stacked on top of Arduino Uno to facilitate communication between one XBee module fixed on the child's arm and another attached to Arduino Uno.

\section{6) Arduino Ethernet Shield}

Arduino Ethernet shield allows Arduino to connect to the internet. It is based on the Wiznet W5100 ethernet chip. The Wiznet W5100 provides a network IP stack capable of both TCP and UDP. It supports up to 4 simultaneous socket connections [29]. Using long wire-wrap headers ethernet shield is connected to the Arduino board which keeps the pin layout intact and allows other shields to be stacked on top. The shield assigned a MAC address and a fixed IP address using Ethernet. Valid IP addresses depend on the configuration of the network. It is possible to use DHCP to dynamically assign an IP to the shield.

An Ethernet shield will be stacked on top of Arduino Uno to provide internet connectivity as data acquired from sensors and transmitted by means of XBee modules is to be transferred over internet to the mobile of the child's guardian. The shield will be connected to a $\mathrm{Wi}-\mathrm{Fi}$ router using standard Ethernet cable (CAT5 of CAT6 with RJ45 connecters).

\section{B. Software Requirements}

An Android mobile application is built to provide a proper alarm mechanism. Android is a Linux based operating system designed primarily for touchscreen mobile devices such as smartphones and tablet computers [30].

Android powered devices are penetrating the smartphone and tablet devices market and the popularity of this open source platform is steadily rising. Therefore, potential customers are likely to have smartphones; which contributes to decreasing the overall cost of the system.

Being an open source platform, Android provides the advantage of making use of free app development tools and technologies that enables us to create a compelling Android based mobile application to meet customer's demands.

\section{System Integration}

As presented in the system flowchart in Figure 2, Temperature and motion sensors are to be interfaced with a microprocessor in which temperature readings will be compared with a threshold. Temperature readings as well as an alarm signal will be transmitted to the mobile phone of the child's guardian if such readings exceeded the predefined threshold. To insure accuracy three successive readings exceeding the threshold will be required to trigger the alarm process.

\section{1) Thermistor Calibration}

Steinhart-Hart calibration equation is used to characterize the nonlinear response of MF51E103e3950 NTC ther- 
mistor by relating the thermistor variable resistance to temperature.

Finding good Steinhart-Hart equation coefficients plays a main role in determining the device's accuracy. We have developed a system of three equations by using three resistance values of the thermistor obtained at three different temperatures provided by the sensor's datasheet as shown in Table 5.

Celsius to Kelvin unit conversion is needed prior to substituting the table values into Steinhart - Hart equation as this model gives the absolute temperature of an NTC thermistor in Kelvin Celsius-Kelvin conversion is done by the equation 2 and the results are shown in Table 6.

$$
\mathrm{Tk}=\mathrm{Tc}+237.15 \rightarrow \quad(\text { Equation } 2)
$$

After converting temperatures from Celsius to Kelvin and after substituting datasheet values into equation 1 the following equations $(3,4,5)$ are developed.

$$
\begin{aligned}
\frac{1}{253.15}=\mathrm{A}+\mathrm{B} & \times \ln (98.99 \mathrm{k})+\mathrm{c} \times \ln ^{3}(98.99 \mathrm{k}) \\
\rightarrow & (\text { Equation } 3) \\
\frac{1}{298.15}=\mathrm{A} & +\mathrm{B} \times \ln (10 \mathrm{k})+\mathrm{c} \times \ln ^{3}(10 \mathrm{k}) \\
& \rightarrow(\text { Equation } 4) \\
\frac{1}{323.15}=\mathrm{A}+\mathrm{B} & \times \ln (3.588 \mathrm{k})+\mathrm{c} \times \ln ^{3}(3.588 \mathrm{k}) \\
\rightarrow & (\text { Equation } 5)
\end{aligned}
$$

After solving the three equations, the coefficients values were found to be as shown in table 7 .

\section{2) System Circuits}

The core temperature-measuring device constantly monitors and analyzes the child's temperature measurements in order to detect any concerning trends. Data processing and analyzing is done locally in the device itself and in the base station after transmitting the collected data using wireless communication.

The device's circuit as shown in Figure 8 is composed of the following components in Table 8:

\section{3) Monitoring Base Station}

The base station receives temperature values sent by the wearable device and performs additional data processing in order to issue an alarm in the case of any abnormal temperature measurements. The System's base station mainly consists of the following components as listed in table 9. Figure 9 shows how these components are connected together in order to perform the required functionality.

The receiver XBee module receives temperature values transmitted by the wearable device and writes these values on the serial port of the microcontroller that is fixed on the Arduino board. In order to allow writing on the serial port; Din and Dout of the XBee are connected with Tx and Rx pins of the microcontroller respectively.

\section{Main Subsystems}

In order to achieve the required objectives, the main circuit of the monitor device and the base station was divided into four subsystems that perform different functionalities as shown in figure 10 .

Each subsystem was built, configured and tested as a single component to assure that the integration of these cooperating subsystems is able to deliver the required overall functionality.

\section{1) Data Collecting And Processing Subsystem}

To read skin temperature measured by MF51E103e3950 thermistor and send it to analog input 1 (Pin 24) of ATmega328 microcontroller is the main function of data collecting subsystem. Converting MF51E variable resistance to voltage value by using voltage divider circuit is required because ATmega328 microcontroller analog pins read voltage values only. This circuit is composed of $10 \mathrm{~K} \Omega$ resistor connected in series with the thermistor.

Resistance-Voltage conversion is done by using the following voltage divider equation:

$$
\mathrm{V}_{\text {out }}=\frac{\mathrm{R}_{\mathrm{s}}}{\mathrm{R}_{\mathrm{s}}+\mathrm{R}_{\mathrm{ntc}}} \times \mathrm{V}_{\text {in }} \rightarrow \text { (Equation 6) }
$$

Where;

Vout is the thermistor output fed into ATmega328 ana$\log$ pin 1

Vin is the 5 volts input voltage

Rs is the series $10 \mathrm{~K} \Omega$ resistor

Rntc is the thermistor resistance which changes according to the change of temperature

ATmega328 microcontroller reads the skin temperature each 10 seconds based on medical research results which state that human body temperature needs at least 10 seconds in order to change its value.

ATmega328 microcontroller is the core component of data processing subsystem. We have used Arduino IDE software to program it with a sketch that implements different functions allowing the microcontroller to provide the required functionality.

Two microcontrollers are used as shown in figure 11; one microcontroller performs data processing on the monitor device and the second microcontroller is fixed on the Arduino Uno board in the base station.

The first microcontroller reads the output voltage of the thermistor and then implements several equations in order to map voltage values to final temperature values. The following line of code is used to read the voltage value from the microcontroller's analog pin:

Vout $=$ analogRead(Analog_Pin)

Where Analog_Pin $=1$

Voltage-temperature mapping first requires converting each voltage value to its corresponding resistance value (Rntc). Re-arranging equation 6 results in the following formula that uses thermistor output voltage fed into pin 1 as an input to calculate thermistor Rntc value.

$$
\mathrm{R}_{\mathrm{ntc}}=\mathrm{R}_{\mathrm{s}}\left(\frac{\mathrm{V}_{\text {in }}}{\mathrm{V}_{\text {out }}}-1\right) \quad \rightarrow \quad \text { (Equation 7) }
$$

Once the final temperature values are calculated, the monitor device's microcontroller sends temperature values to the serial port through Serial.println() function.

Temperature values are wirelessly transmitted from the first microcontroller to the second one through two XBee modules. Once temperature values are ready in the two microcontrollers, determining whether a fever is present is the next task.

Temperature measurements are compared with a threshold value in order to issue an alarm if the temperature value exceeds threshold value. Medical research has determined that a fever is present when armpit temperature 
exceeds $37.3^{\circ} \mathrm{C}$ so the alarm is turned on when temperature measurements exceeds this threshold.

Because accuracy of temperature readings is of great importance, the average value of every three consequent temperatures is calculated and compared with the threshold value in order to increase system accuracy and to avoid false alarms.

\section{2) Alarming Subsystem}

The system is designed to issue multiple alarms at three different places; at the monitor device, at the base station and at the child's guard mobile. Two 8 ohms 1 watt Speakers are used to generate alarms at the first two places.

Speakers are connected to pin 16 (digital pin 10) of ATmega328 microcontroller through $100 \mathrm{ohm}$ resistor in order to play alarm melody using a range of audible pitches.

Three different alarm melodies are used for three different temperature ranges as shown in figure 12. Each melody indicates that the child's temperature falls in a specific range of temperatures so the child's guard can use the melody type as an indicator to his/her child's situation.

Alarms is manually turned off using push buttons that are connected to digital pin 2 of the microcontrollers through $10 \mathrm{~K} \Omega$ resistor.

\section{3) Data Transmission Subsystem}

A wireless XBee module (Sender XBee module) that is fixed on the monitor device is used for transmitting the calculated temperature values to another XBee module (Receiver XBee module) connected with the base station.

Each XBee is serially interfaced with the microcontroller by connecting $\mathrm{Rx}$ and $\mathrm{Tx}$ pins of the microcontroller with Dout and Din pins of the XBee respectively.

XBEE Modules use standard AT commands. These commands allow one to edit the registers found inside the XBEE. Each register has a name; prefixed by AT, such as ATBD which is the Baud Rate register.

XBEE radios only operate at a given Baud Rate; this is the number of bits per second that an XBEE can send. The available Baud Rate and corresponding ATBD value are:

2400 bps, 4800 bps, 9600 bps, 38400 bps, 57600 bps and $115200 \mathrm{bps}$. Both of the XBEEs we used are configured to a Baud Rate of 9600 bps.

The next parameter of interest is the Personal Area Network ID. Only modules with matching PAN IDs can communicate with each other. Unique PAN IDs enable control of which packets are received by a module. The PAN ID is stored in ATID.

Each XBEE Module was assigned an address, denoted by ATMY as well as a destination address, denoted ATDL. If a module's DH was set to 0 and its DL is less than $0 \mathrm{xFFFF}$, data transmitted by that module will be received by any module whose 16-bit address MY parameter equals DL. XBEEs setup is shown in table 10:

An XBEE USB Explorer was used to connect XBEE Modules to the PC. It is a compact circuit board that already has a $3.3 \mathrm{~V}$ regulator; it draws power directly from the serial port.

MaxStream distributes a software called X-CTU which allows one to set all parameters described above, using a Graphical User Interface.

\section{4) Sending Temperature Readings To A Local Server}

Once Temperature Readings are received by the Arduino Uno an HTTP request message is formulated and sent to a script on a Local Server (Wamp Server) using an Ethernet Shield. Temperature Readings included in the HTTP message are then stored in a MYSQL Database. Figure 13 gives further insight on how data flows through the system.

\section{5) Software Implementation}

Users are given the option to monitor their children's body temperature either from their mobile devices or from a website.

An android mobile application is built to monitor children's core body temperature and alarm their guardians in case of an unusual increase in core body temperature.

A Graphical User Interface (GUI) is provided for the children's guardians on their mobile phones to monitor their children's body temperature as he/she goes about their normal daily routine.

A well-designed interface supported with a clear set of commands that can be used to freely navigate through all portions of the application, while simultaneously guiding the user and protecting him/her from his/her own unintentional mistakes.

A simple and efficient to fill out login form appears once the user launches the application. Remembering an email address is easier than remembering a user name. User names can be unwieldy; therefore, users are required to login in with their email address as well as a password.

In order create a new account a typical signup form asks the user to create a user name and a password and to enter his/her email to be used later for login. On a successful login the user is navigated to an interface in which his/her children are displayed. The user is provided with the capability to add/modify a child's information including: their Age and whether or not they experienced a seizure before. The user must then select a child to monitor his/her body temperature. $\mathrm{He} / \mathrm{she}$ is then directed to an interface that supplies time stamped temperature values; an alarm will start beeping intuiting that the temperature exceeded the normal. The user is given the option to turn the alarm off. Figure 14 show snapshot of the GUI for sequence mentioned.

\section{6) Database Design}

The Relationship-Entity model for the database is shown in Figure 15. The database has three tables Users, Children and Temperatures. The first table stores Username, Password and Email of a user required to restrict access to those authorized. The second table stores all child related information including: Experienced Before and Family History; Boolean attributes to indicate whether or not the child experienced a seizure before and if he/she has a family history of febrile seizure.

To pull data together in meaningful ways; the Primary Key in Users table (UserID) is linked to an identically named field in Children table known as a Foreign Key. Similarly, the Primary Key in Children table (ChildID) is linked to an identically named field in Temperatures table known as a Foreign Key.

PhpMyAdmin tool is used to create the database and its tables. 


\section{7) Fetching Data From The Server}

The Android Application on the devices makes an HTTP request with basic HTTP Key and Value parameters. PHP Application Programming Interface (API) classes then accept HTTP requests, interact with PHP classes to get data from or store in database and finally give output in JSON format.

DefaultHttpClient() is used to create an HTTP Client and HttpPost is used to create an HTTP

Post with the URL to Post data to supplied as a parameter.

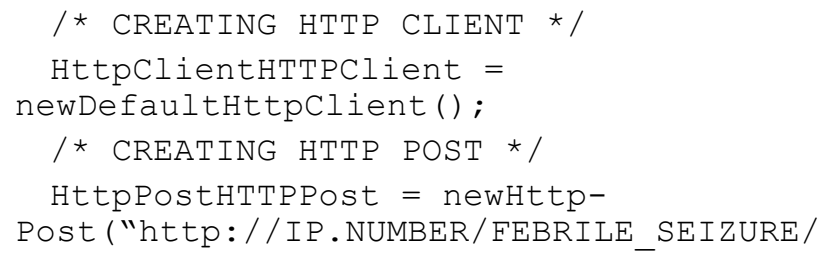

Where http://IP.NUMBER/FEBRILE_SEIZURE/ is the URL on the local Wamp server to post data to.

HTTP Post key and value parameters are formulated using:

List $<$ NameValuePair $>$ Parameters = newArrayList<NameValuePair> (2);

Parame-

ters.add (newBasicNameValuePair("EMAIL", "user(gmail.com")) ;

Parame-

ters.add (newBasicNameValuePair("PASSWOR D", "encrypted_password") ) ;

Key and value Post parameters are then encoded in order to convert all string data into valid URL format using: HTTP-

Post. setEntity (newUrlEncodedFormEntity ( Parameters));

Finally HTTPPost is executed using HTTPClient created before:

HTTPResponse Response = HTTPCli-

ent.execute (HTTPPost);

JSON is a language independent lightweight data interchange format. JSON responses generated by PHP API classes are encoded using:

json_encode (\$RESPONSE);

Website:

A simple easy to use webpage created using HTML. The user is required to enter his/her ChildID in an input field to be able to use the system.

\section{CONCLUSION AND FUTURE WORK}

The proposed system is a low power, reliable, nonintrusive temperature monitor system that processes data acquired from sensors designated to monitor body temperature; an alarm will be issued to the mobile phone of the child's guardian so as temperature is not to exceed a certain limit to prevent febrile convulsions.

With the addition of motion sensor; the system is to be expanded to serve not only as an effective prevention but also an appropriate detection mechanism. Temperature readings will be transmitted to a server instead of a mobile phone for further analysis. Thus, the system will help generate parameters required for medical research purposes including: temperature at which febrile seizure occurs as well as the duration of febrile convulsions. Recent studies tend to find linkage between febrile convulsions and epilepsy [31]. Therefore, it is important to provide a system that constantly monitors children during a febrile seizure to supply parameters required for medical research.

Children with a family history of febrile seizures or have experienced one seizure before as well as those in their first 24 hours of illness are more likely to have febrile convulsions. Therefore, such children will be assigned higher priorities when profiled for medical research.

\section{REFERENCES}

[1] M. Fishman. Patient Information: Febrile Seizures (Beyond the Basics).February 7, 2011. [Online]. [Accessed September 25, 2012]. http://www.uptodate.com/contents/febrile-seizures-beyondthe-basics

[2] K. Raye. The Disadvantages of a Digital Thermometer. [Online]. [Accessed November 5, 2012]. http://www.ehow.com/list_758 0814 disadvantages-digital-thermometer.html - ixzz2BNPNLNWp

[3] C.Lim, C.Byrne, J.Lee. Human Thermoregulation and measurement of Body Temperature in Exercise and Clinical Settings.AnnAcad Med Singapore April 2008.

[4] Wireless Body Thermometer. Mesure Technology Co., Ltd. October 29, 2012.

[5] D. Beasley, J. Bluck, R. Spector. NASA Device Monitors Health of Scientists and Explorers. June 15, 2004.

[6] Spot Vital Signs LXi. October 29, 2012. http://www.welchallyn.com/apps/products/product.jsp?id=11-ac100-0000000001118

[7] Source: Agilent Technologies. Practical Temperature Measurements, Application Note 290, March 2008.

[8] J. Fraden. Handbook of Modern Sensors.Springer-Verlag New York, Inc. 2004.

[9] Vishay Dale .PTC and NTC Thermistors. Document Number 33016. 2002.

[10] PTC Thermistors Applications. Spectrum Sensors and Controls, Inc. 2003

[11] NTC Thermistor Applications. Thinking Electronic Industrial Co., LTD. 2012.

[12] Bowthorpe Thermometrics, Thermometrics Inc, Keystone Thermometrics Corporation. ntc Thermistors.

[13] EPCOS AG. NTC Thermistors General Technical Information.2009

[14] Measurement Specialties, Inc. Model 427 ReusableSkin - Surface Probe. 2012

[15] General Electric. NTC Thermistors: Type MA.

[16] Cantherm. MF51E - High Precision NTC Thermistors for Extremely Accurate Temperature Measurements. 2006.

[17] Quality Thermistor, Inc. NTC Thermistor Design Guide for Discrete Components and Probes. 2007

[18] B. Baker. Thermistors in Single Supply Temperature Sensing Circuits. 1999.

[19] Source: Thermistor Mathematics. July 2011. [Online]. [Accessed December 20, 2012]. http://mathscinotes.wordpress.com/ 2011/07/22/thermistor-mathematics/

[20] G. Recktenwald.Thermistor Calibration.2010

[21] A KYOCERA GROUP COMPANYAVX NTC Thermistors. 2010.

[22] Earthshine Electronics. Arduino Starters Kit Manual - a Complete Beginners Guide to Arduino. 2009.

[23] Arduino Buying Guide. [Online]. [Accessed November 26, 2012]. https://www.sparkfun.com/arduino guide

[24] M. Banzi. Getting Started with Arduino. 2010.

[25] RF Modules Utilizing the ZigBee PRO Feature Set. [Online]. [Accessed November 25, 2012]. http://www.digi.com/products/ wireless-wired-embedded-solutions/zigbee-rf-modules/zigbeemesh-module/xbee-zb-module\#overview 
PAPER

FEBRILE SEIZURE DETECT AND AlARM System

[26] M. Hebel, G. Bricker, D. Harris. Getting Started with XBee RF ModulesA Tutorial for BASIC Stamp and Propeller Microcontrollers. 2010

[27] Parallax, Inc. XBEE Modules, Adapters and Kits. 2010.

[28] RadioSpares. Shield - XBee RF Module. 2009.

[29] Arduino. Arduino Ethernet Shield.2010.

[30] M. Murphy. The Busy Coder's Guide to Android Development. CommonsWare, LLC. 2008.

[31] MRI and EEG Could Identify Children at Risk for Epilepsy after Febrile Seizures. [Online]. [Accessed November 7, 2012] http://www.sciencedaily.com/releases/2012/11/121107161549.htm
[32] Palestinian Central Bureau of Statistics. National Health Accounts 2000 - 2008. January, 2011.

\section{AUTHORS}

Khader Mohammad, Sundous Hussein, and Rawan Rimawi are with the Department of Computer Systems Engineering, Birzeit University, Ramallah, Palestine (emails: Hajkhader@gmail.com, h.sundous@gmail.com, rawanrimawi@googlemail.com).

Submitted 15 December 2014. Published as resubmitted by the authors 25 November 2015. 